\title{
The Broken Health Information Technology Innovation Pipeline: A Perspective from the NODE Health Consortium
}

\author{
Sonya Makhni ${ }^{a} \quad$ Ashish Atreja $^{a} \quad$ Amy Sheon $^{b}$ Brian Van Winkle ${ }^{c}$ \\ John Sharp ${ }^{d}$ Neil Carpenter ${ }^{c}$ \\ a Icahn School of Medicine at Mount Sinai, New York, NY, USA; ${ }^{b}$ Case Western Reserve \\ University, Cleveland, OH, USA; ' ${ }^{\circ}$ LifeBridge Health, Baltimore, MD, USA; ${ }^{d}$ Kent State \\ University, Kent, OH, USA
}

Keywords
Health information technology $\cdot$ Innovation · Digital health · Outcomes

\section{Abstract}

Background: The Network of Digital Evidence (NODE) was formed to further advance the field of health information technology (HIT) and evidence-based digital medicine at different healthcare institutions nationwide. As the NODE network reviewed the state of the field, it was noted that despite substantial financial and human capital investments, the processes and results of HIT innovation seem chaotic and subpar, especially in comparison to the more wellestablished drug and device industries. During the course of this white paper, we will explore the causes for this observed phenomenon as well as propose possible solutions to improve the state of HIT. Methods: We compared the entire process of discovery, proof of concept, Food and Drug Administration (FDA) review, and postmarket monitoring and distribution/ implementation of HIT innovations to the equivalent processes for drugs and devices. Whereas drug and device innovations are subject to a standardized pipeline of production, HIT innovations are not held to equivalent standards. Conclusions: As a result, HIT lags behind the more mature drug and device industries in producing effective and reliable products. This leads to an inefficient use of already scarce healthcare resources. The authors believe that the HIT industry must adopt many of the mechanisms implemented by the drug and device industries as dictated by their innovation pipelines of discovery, proof of concept, FDA review, and postmarket monitoring and distribution/implementation. We propose an eight-point plan to fundamentally evolve the HIT lifecycle, including reforms for institutions such as neu- 
Makhni et al.: The Broken Health Information Technology Innovation Pipeline:

A Perspective from the NODE Health Consortium

tral government agencies, new health system boards and management systems, modified incentive structures, improved relationships with financial investors and start-ups, patient engagement, and enhanced mechanisms to improve HIT adoption.

(c) 2017 The Author(s)

Published by S. Karger AG, Basel

\section{Introduction}

When it comes to health information technology (HIT), innovative ideas and companies abound. However, downstream improvements in outcomes or costs of care resulting from these innovations have to date been underwhelming. There is no better example to illustrate this observation than the limited results yielded by the massive investment in electronic health records (EHRs) [1]. A more recent example can be found in the consumer USD 28.7 billion wearable technology market [2]. A recent randomized controlled clinical trial found that individuals using wearables lost less weight over 24 months than those making lifestyle changes alone [3]. An unwieldy HIT ecosystem, especially in digital medicine, has developed, one in which innovators create products and then scramble to navigate the complex and opaque needs and buying processes of health systems, and medical institutions struggle to sort through the vapor and correctly identify the legitimate and promising innovations. Compounding this issue is the lack of patient engagement during the innovation lifecycle, which prevents products from achieving their full impact.

Some would attribute this underperformance to the general attributes of the healthcare sector, but we, as members of the Network of Digital Evidence (NODE) [4], disagree. One needs only to look to the drug and device industries to see examples of far more mature and effective, albeit imperfect, processes that have guided countless innovations from development to scale and implementation.

For example, in 2016 alone, 22 novel drugs and 26 novel devices were approved by the Food and Drug Administration (FDA) for use as both safe and effective [5, 6]. A simple internet search will yield all pertinent information related to the drugs or devices, as well as which ones may have been recalled. The end consumers may not have heard of a particular innovation, but they can be assured that the drug or device in question has passed a rigorous FDA-led evaluation. In fact, failures of devices and drugs become major news stories. The Morcellator surgical device, used in the removal of tissue during laparoscopic procedures, is a recent example. The possibility of the device spreading cancer cells received a substantial amount of press and discussion as a result [7].

Compounding this noise is the fact that healthcare providers themselves are unclear as to which HIT innovations they should prioritize as valuable. They are inundated with dozens of "Top 10 Innovations in HIT" lists, almost none of which are supported by peer-reviewed studies. The industry is saturated with cherry-picked and massaged data whose quality and relevance ranges widely.

Nevertheless, there is widespread acknowledgment of the importance of HIT advancement. Almost everyone in the industry thinks we need better HIT - and the advancement of IT in other industries is widely credited with the productivity boom in the 1990s. It took years for the investments in the 1980s to be translated into productivity in the 1990s - and that might be true again with HIT investment over the past decade.

Recent legislation is evidence enough to highlight that HIT advancement needs to be a priority. In 2009, for example, the Health Information Technology for Economic and Clinical Health (HITECH) Act paved the way for the rapid expansion of EHR companies, and the Medicare Access and CHIP Reauthorization Act (MACRA) final ruling in 2016 essentially required physicians to increase their use of certified EHR technologies [8]. These policies 


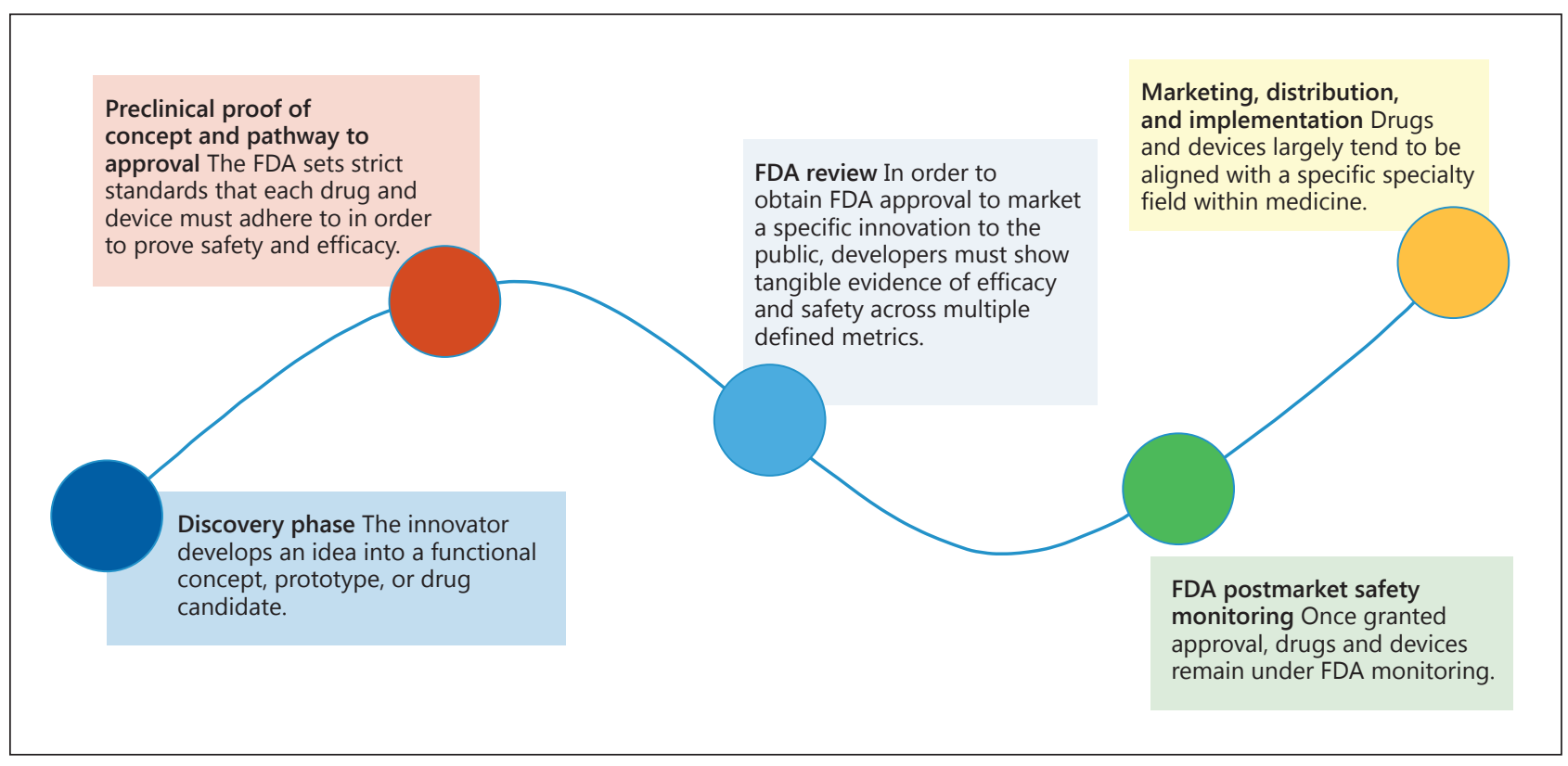

Fig. 1. The Food and Drug Administration (FDA) drug and discovery innovation pipeline.

reflect the hope that cost savings, quality improvement, and better health will result from reliable, interoperable IT systems.

Despite major investments, drug and device development appear to dramatically outpace that within HIT, but why?

One answer ultimately comes down to the maturity of the innovation pipeline across those three segments of the healthcare system. The drug and device pipeline is far more standardized, academic, and regulated than that for HIT, and it is this level of sophistication that enables a consistent production of novel drugs and devices. It is clear that HIT must develop a comparably evolved pipeline; only then will this sector stand a chance of enjoying a steady, reliable stream of implementable innovation. Our paper outlines a roadmap to this desired future state.

\section{The FDA Drug and Device Innovation Pipeline}

With respect to drugs and devices, the FDA has established a very clear, regulated process that drives innovations from development to testing, approval, and surveillance [9]. This pipeline can be generalized as follows (Fig. 1).

\section{Discovery Phase}

The innovator develops an idea into a functional concept, prototype, or drug candidate. Research institutions and pharmaceutical companies ensure sustained innovation through directed efforts to drive and promote innovation via various incentive schemes, cultural norms, and infrastructure supports. Community-based participatory research approaches ensure representation of diverse viewpoints at the earliest stages of defining the questions of interest for research. 
Makhni et al.: The Broken Health Information Technology Innovation Pipeline:

A Perspective from the NODE Health Consortium

\section{Preclinical Proof of Concept and Pathway to Approval}

The FDA sets strict standards that each drug and device must adhere to in order to prove its safety and efficacy. Drugs are required to follow a set series of preclinical and clinical studies, and devices are subject to specific controls and studies that are determined by each innovation's risk profile. The FDA regulates all aspects, including study design, personnel and facilities, and quality assurance.

\section{FDA Review}

To obtain FDA approval to market a specific innovation to the public, developers must show tangible evidence of efficacy and safety across multiple defined metrics. All submissions are reviewed within a designated time frame by a team comprised of specialized members; this body can consult an independent advisory committee for additional expertise, if needed.

\section{FDA Postmarket Safety Monitoring}

Once granted approval, drugs and devices remain under FDA monitoring. Developers are required to continuously report adverse events, updates, and changes, and they are subject to periodic inspections and reviews. This is to ensure continued efficacy and safety of the marketed device and/or drug.

\section{Marketing, Distribution, and Implementation}

Drugs and devices largely tend to be aligned with a specific specialty field within medicine. Developers target synergistic societies and partners within the relevant discipline and educate the target market of the device/drug. This occurs through multiple forums, such as conferences, peer-reviewed literature, demonstrations, and direct-to-physician advertising and communication. Key decision makers are the gatekeepers to new innovations, and drug/ device firms are well informed as to who these individuals are at any given institution.

\section{The Broken HIT Innovation Pipeline}

To better understand why innovation within HIT trails behind its true potential, we need only look to this pipeline to find multiple points of fracture (Fig. 2).

\section{The Breakdown of the Discovery Phase: An Incomplete Innovation Culture}

"Innovation culture" is a broad term and can refer to a multitude of factors. However, with respect to HIT, we feel that two factors are the most important in informing this breakdown of an innovation culture.

A Culture of Respect for IT. The first factor is respect. During an HIT summit meeting attended by some of the top leaders within the field, a presenter was asked the question, "When do you think someone will become chair of a major academic medicine institute because of their pioneering efforts in HIT?" In response, the room broke out in laughter, and the speaker from an academic medical center just chuckled. This anecdote is quite representative of how HIT is viewed by the classic academics within medicine, a field in which the very currency of success lies in research studies, device patents, and pharmacological discovery. Incentives are aligned with production within these spaces and not within HIT. Yet, we are already well aware of the profound importance HIT has on the general functioning and potential cost saving effects innovations within this space can have.

A Well-Defined Definition of Need. The next major cultural break point has to do with sense of need. The drug and device market has a well-established sense of need. Walk into any health system and ask a physician what new drugs or devices he/she needs from industry, 


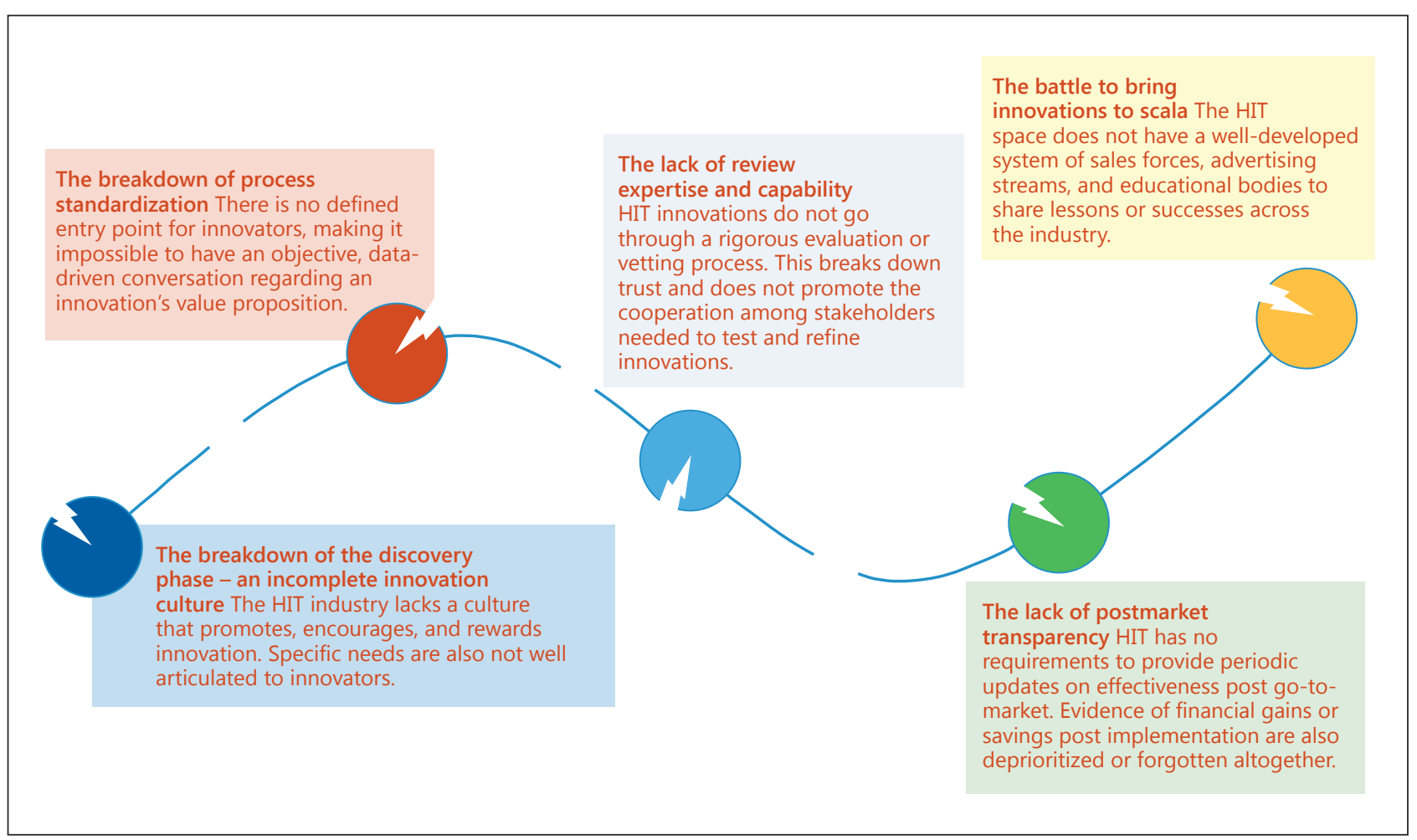

Fig. 2. The broken health information technology innovation pipeline. HIT, health information technology.

and you will hear a list of items that likely resonate with many other physicians within that specialty. Now try that with HIT. You would likely hear a long list of general terms, such as "more interoperability," "easier to use," "better outcome measures," but it is far more difficult to articulate what specific innovations are necessary to address these very valid but somewhat vague needs. Patient perspectives are also surely underrepresented at this earliest phase.

\section{The Breakdown of Process Standardization}

Drugs and devices are forced through a well-defined process that requires studies, trials, inspections, and study reports that often fit into specialist and subspecialist peer-reviewed journals and conferences to debate safety and efficacy. In contrast, HIT has no defined entry point to the health industry or even well-known, published, and independent industry experts who are not employed by HIT vendors to distinguish HIT fact from fiction. This makes it impossible to have an objective data-driven, industry-focused conversation about the value proposition of different innovations. As a result, investors and innovators have difficulty trusting a "review-less" system.

\section{The Lack of Review Expertise and Capability}

The drug and device review process is also led by FDA reviewers - who are specialized in areas such as study design and statistical analyses - enabling them to thoroughly and capably evaluate an innovation. HIT innovation firms generally have neither the vetting process nor the in-house expertise to implement rigorous evaluation programs. As a result, end consumers, be they health systems or individual patients, must rely on the (frequently unsubstantiated) claims made by vendors. Since these claims do not often hold up, healthcare 
Makhni et al.: The Broken Health Information Technology Innovation Pipeline:

A Perspective from the NODE Health Consortium

institutions have become wary of HIT innovators. Institutions are also prone to allowing small-scale HIT innovations to appease some physicians - rather than having HIT be a central component of the organization's strategy, with a plan to scale those innovations. Ultimately, this weariness does not promote the kinds of cooperative and reliable partnerships that inventors, evaluators, and implementers need to test, refine, and implement HIT innovations.

\section{The Lack of Postmarket Transparency}

We see at least two distinct issues with postmarket monitoring.

First, whereas drugs and devices are required to provide periodic updates to the FDA, no such requirement is in place within the HIT space. As such, vendors lack incentives to provide data that may be unfavorable. And unlike the drug and device arenas, there are almost no honest forums where system or patient-level consumers can report adverse or unintended events. Professional HIT-related events are heavily dominated by vendors, lacking the type of boundaries widely accepted for events relating to drugs and devices. Today the primary mechanism by which health systems are informed of another health system's failed projects is when a senior individual is fired, or if a financial target falls short enough to become a news story.

Second, HIT innovators rarely articulate clear and validated financial savings as part of their pitch due to limited experience in health system or payor finances. Meanwhile, healthcare systems can be unwilling or unable to substantiate these saving themselves. They often lack needed claims data, variable costing information, or the ability to navigate the large data sets and complex accounting issues. Acquiring these capabilities can represent large investments for a health system. Compounding this issue is the tendency for providers to work around "finance" when implementing projects. At our institutions it is not terribly uncommon for us to review a project, notice the lack of financial expertise on the team, and temporally pause the project to engage finance before continuing.

Underlying this dynamic is that unlike many other industries, healthcare can be uncomfortable or underprepared to integrate tight economic management into core functions of the organization. This is for two reasons, the first and foremost being the literal life and death implications of some healthcare delivery choices, as well as the emotional nature of the larger public discussions around many healthcare topics (e.g., end of life decisions). Second, many nonfinance stakeholders tend to see healthcare as an engine of ever expanding jobs and incomes. For example, when, during a large NODE event, one of the authors mentioned fulltime equivalent reduction or reallocation as a central yet often missing element of new IT business cases, the room gasped in shock. The reaction would have otherwise been surprising, if not for the healthcare context, given that a reduction in full-time equivalents is typically fundamental to IT business cases in other industries. In most other industries, technology tends to remove positions, not to create them.

Finally, the prohibition against measuring cost-effectiveness in studies funded by the Patient-Centered Outcomes Research Institute (PCORI) is also not helpful in this regard.

\section{The Battle to Bring Innovations to Scale}

Once device or drug solutions become "green-lighted" by the FDA, companies tap into physician subspecialty committees and clinical guidelines regarding distribution and scaling. The solutions also use well-developed system of sales forces, advertising streams, and educational bodies in order to share knowledge of the new innovation with the relevant players across the country.

The HIT space lacks an analogue, and the distribution channels that do exist are less streamlined and even quite chaotic. At the first level, it is not always clear who the relevant key decision maker is for a given innovation. For example, a program to improve operational 
Makhni et al.: The Broken Health Information Technology Innovation Pipeline:

A Perspective from the NODE Health Consortium

efficiency of operating rooms may have been "green-lighted" by the IT department, but the relevant decision maker within the department of surgery may be less clear or even unsupportive of the measure, or vice versa for that matter. This lack of clarity makes targeted education-based marketing far more decentralized and ineffective. Finally, information dissemination tends to lack the scientific rigor we have come to expect from a new drug or device. For example, if you wander the floor of the Healthcare Information and Management Systems Society (HIMSS), thousands of vendor booths offer displays replete with flashy visual aids and giveaways, but little evidence provided in the form of conventional posters and papers that are required currency at traditional clinical conferences.

\section{Fixing the Broken HIT Pipeline: An Eight-Point Plan}

It is clear that the HIT pipeline is fractured at multiple points, but it is also true that HIT is an immature industry. HIT can transform over time to become a key solution in solving the healthcare productivity crisis plaguing this country. However, for this to be accomplished, the industry has to change. Below are some steps we feel lay out the beginning of that industry transformation.

\section{Regulatory and Third-Party Monitoring}

(1) Creation of a neutral, third-party body or coordination among existing units to become a quasi-FDA for HIT, responsible for mandating approval for government reimbursement of new HIT and for enforcing adherence to the review and reporting processes. Private payors can experiment at will, but the Centers for Medicare \& Medicaid Services (CMS) will set a baseline threshold which all innovations must pass.

(2) Establishment of a contract resource organization for firms looking to prove their software's value. The contract resource organization will have a function within HIT similar to those within the drugs and devices industries. It will define clear and consistent outcome measures within HIT, articulate a "HIT value proposition," and create tools to enable and require sharing of all outcomes, regardless of results.

(3) Journal editors could establish consistent reporting standards, including mandatory registration of clinical trials prior to implementation.

\section{Institutional Alignment of Relevant Bodies}

(4) Increase the visibility of HIT to health system boards and other key stakeholders. The reality is that many leaders know little about HIT, despite the large amounts of capital investments that are being directed towards this space. Health system boards need greater IT expertise and education surrounding IT. HIT may not be as "exciting" to donors or board members, but it is critical to the future of healthcare. HIT champions need to convey and effectively communicate the HIT value proposition to institutional leadership. The receipt of a national HIT award to one of our affiliated institutions appears to be a well-kept secret, even among clinical leaders at that institution.

(5) Build formal structures in order to establish key decision makers and formal evaluation criteria as well as to operationalize innovations. Payors and health systems should be encouraged to publish how their HIT investments are made and who the relevant key decision makers are in each relevant department. Health systems should not invest in HIT projects without concrete plans and resources necessary to scale the innovation. Institutions should consider utilizing "implementation councils" that are dedicated to operationalizing an approved HIT intervention. 
Makhni et al.: The Broken Health Information Technology Innovation Pipeline:

A Perspective from the NODE Health Consortium

(6) Greater professionalization of HIT. (a) Institute formalized support structures, such as incentives for protected time, resources, promotions, and training to increase HIT innovation from within health systems - e.g., evolve the CART model (clinical, administration, research, and teaching) for physician compensation to the CARTT model (with technology as a new and distinct category of support). (b) Introduce formalized training in HIT finance, economies, study design, strategy, operations, etc. into graduate medical education training. Integrate this training into existing informatics fellowships or as an alternate graduate medical education training program. (c) Recognize HIT pursuits as legitimate pathways for clinicians and scholars seeking tenure or other leadership tracks, just as is done for basic science or translational science research.

(7) Improved relationships with start-ups. (a) Start-ups to partner with health systems to co-develop solutions and implementation plans, as opposed to utilizing the traditional process of having start-ups "pitch" and then "rapid prototype." (b) Financial bodies to extend the lifecycle for investments in order to show well-documented results that can withstand peer review. Fewer but better funded companies would lead to products with better return of investment.

(8) Promotion of HIT to clinicians, physicians, and department leaders. (a) Institutions to encourage and facilitate physician ownership in HIT companies and intellectual property ownership. (b) Clinicians to build appropriate teams with diverse areas of expertise within finance, technology, design, and implementation of solutions.

(9) Co-creating HIT to build better solutions and improve adoption [10]. (a) Institutions to encourage and facilitate physician ownership in HIT companies and intellectual property ownership. These stakeholders know the problems of providers best and need to be involved in solution development and testing. (b) Clinicians to build appropriate teams with diverse areas of expertise within finance, technology, design, and implementation of solutions. (c) Technology innovators to work directly with all stakeholders - including patients, especially those with low socioeconomic status and health status - in designing products that are suitable to diverse patient populations. Patients, patients advocacy organizations, and caregivers should be involved early during design thinking sessions so they can help shape the vision during the ideation stage [11]. In addition, they should be involved in every step of evaluation and research planning, something that is strongly advocated by independent organizations such as the PCORI [12].

These changes will not perfectly solve all of the problems we have discussed in this paper, but are meant to help the industry evolve into a better state. Our proposal is designed to learn from what has worked elsewhere and adapt these lessons to the HIT space. And while some of the solutions will fit, it is likely that some will not. For example, our proposal may incidentally prevent some promising new start-ups from forming or reaching their full potential, for the burden of proof may require more capital than they are able to raise. Alternatively, this proposal may also appropriately prevent a number of "unvalidated" and "unvalidatable" solutions from diverting capital away from solutions that can be legitimately validated. Change will need to be an iterative process, just as in the drug and medical device spaces where stakeholders are constantly weighing the competing values of efficiency, transparency, safety, impact, and cost.

\section{Conclusions}

With a society so focused on innovating and driving positive change, it is indeed an exciting time for healthcare. The not-so-distant future holds promises of cutting-edge developments in such areas as precision medicine, population health, and EHR interoperability 
Makhni et al.: The Broken Health Information Technology Innovation Pipeline:

A Perspective from the NODE Health Consortium

[11]. In fact, the entire healthcare ecosystem stands amidst dramatic changes and developments. It is our hope that a more standardized, regulated pipeline can help optimize the positive impact of these new and exciting innovations for as many people as possible.

\section{Acknowledgements}

We would like to thank and acknowledge all of the leaders in the NODE Health Consortium.

\section{Ethics Statement}

The authors have no ethical conflicts to disclose.

\section{Conflict of Interest Statement}

The authors declare no conflicts of interest.

\section{References}

1 Kellermann AL, Jones SS: What it will take to achieve the as-yet-unfulfilled promises of health information technology. Health Aff (Millwood) 2013;32:63-68.

2 Weinswig D: The Wearables Report: Reviewing a Fast Changing Market. Fung Global Retail and Technology, 2016.

3 Jakicic JM, Davis KK, Rogers RJ, King WC, Marcus MD, Helsel D, Rickman AD, Wahed AS, Belle SH: Effect of wearable technology combined with a lifestyle intervention on long-term weight loss: the IDEA randomized clinical trial. JAMA 2016;316:1161-1171.

4 Network of Digital Medicine in Health (NODE Health). Available at www.nodehealth.org (last accessed April $20,2017)$.

5 Woodcock J: Novel Drugs Summary 2016. U.S. Food and Drug Administration, 2017. Available at www.fda. gov/drugs/developmentapprovalprocess/druginnovation/ucm534863.htm (last accessed April 20, 2017).

6 Medical Devices Cleared or Approved by FDA in 2016. Available at www.fda.gov/MedicalDevices/ProductsandMedicalProcedures/DeviceApprovalsandClearances/Recently-ApprovedDevices/ucm494389.htm (last accessed April 20, 2017).

7 Voelker R: Morcellator risk was known. JAMA 2017;317:1110.

-8 Schneider EC, Hall CJ: Improve quality, control spending, maintain access - can the merit-based incentive payment system deliver? N Engl J Med 2017;376:708-710.

9 Medical Device Innovation Initiative White Paper. U.S. Food and Drug Administration. Available at www.fda. gov/aboutfda/centersoffices/officeofmedicalproductsandtobacco/cdrh/cdrhinnovation/ucm242067.htm (last accessed April 20, 2017).

10 Rudin RS, Bates DW, MacRae C: Accelerating innovation in health IT. N Engl J Med 2016;375:815-817.

11 Liedtka J: Learning to use design thinking tools for successful innovation. Strategy Leadersh 2011;39:13-19.

12 Selby JV, Beal AC, Frank L: The Patient-Centered Outcomes Research Institute (PCORI) national priorities for research and initial research agenda. JAMA 2012;307:1583-1584. 